\title{
COMPLETELY REDUCIBLE OPERATORS THAT COMMUTE WITH COMPACT OPERATORS
}

\author{
SHLOMO ROSENOER
}

\begin{abstract}
It is shown that if $T$ is a completely reducible operator on a Banach space and $T K=K T$, where $K$ is an injective compact operator with a dense range, then $T$ is a scalar type spectral operator. Other related results are also obtained.
\end{abstract}

Let $A$ be an algebra of bounded linear operators on a Banach space $X$. lat $A$ is the lattice of (closed) invariant subspaces of $A$. We say that $A$ is completely reducible if for every $M \in$ lat $A$ there is $N \in$ lat $A$ with $M+N=X$ (that is, $M \cap N=0$ and the algebraic sum $M+N$ coincides with $X$ ). An operator $T$ is completely reducible if the algebra generated by $T$ is. It is unknown whether a weakly closed unital completely reducible algebra must be reflexive; that is, must contain every operator which leaves invariant its invariant subspaces. Some partial solutions of this problem can be found in $[\mathbf{1}, \mathbf{6}, \mathbf{7}]$.

In this paper we show that every completely reducible operator commuting with an injective compact operator with a dense range is a scalar type spectral operator. In particular, the weakly closed unital algebra generated by such an operator must be reflexive. This result seems to be unknown even for operators on a Hilbert space. Also, we show that every compact completely reducible operator must be a scalar type spectral operator. This answers a question raised by E. Azoff and A. Lubin (see the last page of [1]) and, independently, by V. Lomonosov. Finally, our result generalizes the results of Loginov and Sul'man [2] and Rosenthal [5] on reductive Hilbert space operators that commute with compact operators.

The following theorem is the central result of the author's paper [4], where it was stated in a slightly different form:

THEOREM 1. Let $A$ be a commutative operator algebra on a Banach space $X$. If the commutant of $A$ is completely reducible and the ranges of compact operators in $A$ span $X$, then every operator in $A$ is a scalar type spectral operator. If, in addition, $\mathcal{A}$ is a weakly closed unital completely reducible algebra, then $A$ is generated, as a uniformly closed algebra, by a complete totally atomic Boolean algebra of projections. Moreover, $A$ is reflexive and admits spectral synthesis (i.e., every invariant subspace of $A$ is spanned by its one-dimensional invariant subspaces).

Thus, in order to prove the result described above, it suffices to show that if $A$ is a commutative completely reducible algebra which has enough hyperinvariant subspaces, then the commutant of $A$ is also completely reducible. This will be done in Theorem 8 below. The above result then follows easily, a sufficient supply of

Received by the editors September 14, 1981.

1980 Mathematics Subject Classification (1985 Revision). Primary 47B40. 
hyperinvariant subspaces being provided by Lomonsov's theorem. It can be shown, by a slight variation of the proof of Theorem 8 , that the word "hyperinvariant" in its statement can be replaced with "invariant."

Let us introduce some definitions and notation. For Banach spaces $X$ and $Y$, $\mathcal{L}(X, Y)$ denotes the collection of all bounded linear operators from $X$ to $Y ; \mathcal{L}(X, X)$ is denoted by $\mathcal{L}(X) . X^{*}$ means the conjugate space of the Banach space $X$. For $M \subseteq X, M^{\perp}$ is an annihilator of $M$ in $X^{*}$. An operator $E$ in $\mathcal{L}(X)$ is a projection if $E^{2}=E$. If $E$ and $F$ are projections, we write $E \leq F$ provided $E F=F E=E$. Clearly, $E \leq F$ if and only if $E(X) \subseteq F(X)$ and $\operatorname{Ker} E \supseteq \operatorname{Ker} F$. If $E$ is a projection, we write $E^{\perp}$ for $I-E$. If $\mathcal{A}$ is a subalgebra of $\mathcal{L}(X)$, then $\mathcal{A}^{\prime}$ denotes the commutant of $A$; that is, the set of all operators in $\mathcal{L}(X)$ that commute with every operator in $A$. Hyperinvariant subspaces of $A$ are those invariant for $A^{\prime}$. We will write $P(A)$ for the family of all projections in $A^{\prime}$, and $P_{0}(A)$ for the set of those projections in $P(A)$ whose range is hyperinvariant for $A$. Finally, for $E$ in $P(A)$, we define $\operatorname{int}_{E} A$ as the set of all $T \in \mathcal{L}\left(E^{\perp}(X), E(X)\right)$ such that

$$
E A E T=T E^{\perp} A E^{\perp} \quad \text { for each } A \in A
$$

or, equivalently,

$$
(A \mid E(X)) T=T\left(A \mid E^{\perp}(X)\right) \quad \text { for each } A \in A .
$$

Clearly, an operator algebra $A$ is completely reducible if and only if for every $M$ in lat $A$ there is a projection in $P(A)$ with range $M$. Note also that for $A$ completely reducible and $M$ in lat $\mathcal{A}$, the restriction of $A$ to $M, A \mid M$, is also completely reducible.

We shall need some very elementary lemmas. The first is well known.

LEMMA 2. Let $X$ be a Banach space and let $X_{1}$ and $X_{2}$ be subspaces of $X$ with $X_{1}+X_{2}=X$. Then $X$ is isomorphic to the exterior direct sum $X_{1} \oplus X_{2}$ defined as a vector space of ordered pairs $\left(x_{1}, x_{2}\right), x_{i} \in X_{i}$, endowed with the norm $\left\|\left(x_{1}, x_{2}\right)\right\|=\left\|x_{1}\right\|+\left\|x_{2}\right\|$.

LEMMA 3. Let $\mathcal{A}$ be a subalgebra of $\mathcal{L}(X)$ and $E \in \mathcal{P}(\mathcal{A})$. Then $E^{\perp}(X)$ is in lat $\mathcal{A}^{\prime}$ if and only if $\operatorname{int}_{E} \mathcal{A}=0$.

Proof. Suppose $E^{\perp}(X) \in$ lat $\mathcal{A}^{\prime}$. For each $T \in \operatorname{int}_{E} \mathcal{A}, E T E^{\perp}$ is in $\mathcal{A}^{\prime}$, so that $E T E^{\perp}=0$ and $T=0$. Conversely, for each $B \in \mathcal{A}^{\prime}, E B E^{\perp} \mid E^{\perp}(X)$ is in $\operatorname{int}_{E} \mathcal{A}$, and $\operatorname{int}_{E} A=0$ implies $E B E^{\perp}=0$, so that $E^{\perp}(X)$ is invariant under $B$.

LEMMA 4. Let $A$ be a subalgebra of $\mathcal{L}(X)$ and $F \in \mathcal{P}_{0}(A)$. Let $X_{1}=F(X)$ and $X_{2}=\operatorname{Ker} F$.

(i) For each $M \in$ lat $A^{\prime}, F(M) \in$ lat $A^{\prime}$.

(ii) $A$ subspace $Y$ which contains $X_{1}$ belongs to lat $\mathcal{A}^{\prime}$ if and only if $Y=X_{1}+Y_{1}$, where $Y_{1} \subseteq X_{2}$ and $Y_{1} \in \operatorname{lat}\left(\mathcal{A} \mid X_{2}\right)^{\prime}$.

Proof. (i) For each $B \in A^{\prime}, B F(M) \subseteq F(X)$, since $F(X)$ is hyperinvariant, and $B F(M) \subseteq M$, since $M$ is hyperinvariant and $F \in A^{\prime}$. Hence, $B F(M) \subseteq$ $F(X) \cap M=F(M)$.

(ii) Let $Y \supseteq X_{1}$ and $Y \in$ lat $\mathcal{A}^{\prime}$. In particular, $Y$ is invariant under $F$, so that $Y=X_{1}+Y_{1}$ for some $Y_{1} \subseteq X_{2}$. For each $C \in\left(\mathcal{A} \mid X_{2}\right)^{\prime}, F^{\perp} C F^{\perp} \in \mathcal{A}^{\prime}$, and $C\left(Y_{1}\right)=F^{\perp} C F^{\perp}\left(X_{1}+Y_{1}\right) \subseteq F^{\perp}(Y)=Y_{1}$; that is, $Y_{1} \in \operatorname{lat}\left(\mathcal{A} \mid X_{2}\right)^{\prime}$. Conversely, 
if $Y_{1} \in \operatorname{lat}\left(A \mid X_{2}\right)^{\prime}$ and $B \in A^{\prime}$, then $F^{\perp} B F^{\perp} \mid X_{2}$ is in $\left(A \mid X_{2}\right)^{\prime}$ and $F^{\perp} B F=0$. It follows that $B(Y)=\left(F B+F^{\perp} B F^{\perp}\right)(Y) \subseteq X_{1}+Y_{1}=Y$.

The following two lemmas will enable us to reduce the proof of the main result to the case when the completely reducible commutative algebra has no nonzero finite-dimensional invariant subspaces.

LEMMA 5. Let $A \subseteq \mathcal{L}(X)$ be a completely reducible algebra such that the onedimensional subspaces in lat $A$ span $X$. Then $A^{\prime}$ is completely reducible.

Proof. Clearly, $A$ is commutative. We claim that $A$ admits spectral synthesis. Indeed, let $M \in$ lat $\mathcal{A}$. Then there exists $F \in P(A)$ such that $E(X)=M$. Since $E$ transforms every one-dimensional invariant subspace of $A$ into an invariant subspace of $A$ of dimension no greater than one, $E(X)$ is spanned by one-dimensional elements of lat $A$.

Now suppose $X_{1} \in$ lat $\mathscr{A}^{\prime}$. Since $\mathcal{A}^{\prime} \supseteq \mathcal{A}, X_{1}$ is also in lat $\mathcal{A}$, and one can find $X_{2} \in$ lat $\mathcal{A}$ with $X_{1}+X_{2}=X$. We sill show that $X_{2}$ is also in lat $\mathcal{A}^{\prime}$. Suppose not. Denote by $E$ the projection onto $X_{1}$ along $X_{2}$. Then, by Lemma 3 , int ${ }_{E} A \neq 0$, and, by our claim above, there exist such $T \in \operatorname{int}_{E} A$ and one-dimensional $N \in \operatorname{lat}\left(A \mid X_{2}\right)$ such that $M=T(N) \neq 0$. It is very easy to see that $M \in$ lat $A$ and the algebra $A \mid(M+N)$ consists only of multiples of the identity. Denote by $S$ an operator which maps $M$ into $N$ and is identically zero on some invariant complement to $M+N$. Then $S \in A^{\prime}$, but $X_{1}$ is not invariant for $S$, a contradiction.

LEMMA 6. Let $A \subseteq \mathcal{L}(X)$ be a completely reducible algebra. Suppose $X_{1}$ is spanned by all one-dimensional subspaces in lat $\mathcal{A}$ and $X_{2}$ is in lat $\mathcal{A}$ with $X_{1} \dot{+}$ $X_{2}=X$. Then both $X_{1}$ and $X_{2}$ are in lat $A^{\prime}$.

Proof. Obviously, $X_{1}$ lies in lat $\mathcal{A}^{\prime}$. Suppose $X_{2}$ does not. Then, denoting by $E$ a projection onto $X_{1}$ along $X_{2}$, we conclude that $\operatorname{int}_{E} A \neq 0$. Choose nonzero $T \in \operatorname{int}_{E} A$. Since $\operatorname{cl} T\left(X_{2}\right) \in \operatorname{lat}\left(A \mid X_{1}\right)$ and, as has been noted in the proof of the previous lemma, $A \mid X_{1}$ admits spectral synthesis, we can find a one-dimensional $P \in$ $P\left(A \mid X_{1}\right)$ such that $P T \neq 0$. However, $P T \in \operatorname{int}_{E} A$, so that $\operatorname{Ker} P T \in \operatorname{lat}\left(\mathcal{A} \mid X_{2}\right)$. On the other hand, codim Ker $P T=1$ and, since $A \mid X_{2}$ is completely reducible, $\mathcal{A} \mid X_{2}$ has a one-dimensional invariant subspace, which contradicts the definition of $X_{1}$ and therefore completes the proof.

LEMMA 7. Suppose $\mathcal{A} \subseteq \mathcal{L}(X)$ is a commutative completely reducible algebra which has the following property: for every nonzero $M \in$ lat $\mathcal{A}^{\prime}$, there is $N \in$ lat $\mathcal{A}^{\prime}$ such that $N \subseteq M, N \neq 0, N \neq M$. Let $M_{1}, M_{2}, \ldots, M_{n}, \ldots$ be an infinite sequence of nonzero subspaces in lat $\mathcal{A}^{\prime}$. Then there exists such an $F \in \mathcal{P}_{0}(\mathcal{A})$ that $F^{\perp}\left(M_{1}\right) \neq$ 0 and $F\left(M_{n}\right) \neq 0$ for infinitely many $n$.

Proof. Choose hyperinvariant $N \subseteq M_{1}, N \neq 0, N \neq M_{1}$. Since $A^{\prime} \supseteq A$, $N \in$ lat $A$, and there exists $P \in P_{0}(A)$ with range $N$. Now consider two cases.

Case 1. $P\left(M_{n}\right) \neq 0$ for only finitely many $n$.

Then there is an infinite set of positive integers $J$ such that $M_{n} \subseteq P^{\perp}(X)$ for all $n$ in $J$. Let $F$ denote a projection of $P_{0}(A)$ onto a subspace $\bigvee_{n \in J} M_{n}$ such that $F \leq P^{\perp}$. Then $F\left(M_{n}\right)=M_{n} \neq 0$ for every $n \in J$ and $F^{\perp}\left(M_{1}\right) \supseteq N \neq 0$.

Case 2. $P\left(M_{n}\right) \neq 0$ for infinitely many $n$.

Then take $F=P$. Clearly, $F\left(M_{n}\right) \neq 0$ for infinitely many $n$. On the other hand, $F^{\perp}\left(M_{1}\right) \neq 0$, since $N \neq M_{1}$. 
Now we are ready for the proof of our main result.

THEOREM 8. Let $A \subseteq \mathcal{L}(X)$ be a commutative completely reducible algebra. Suppose that for every hyperinvariant subspace $M$ of $A$ of dimension and codimension greater than 1, there exist nontrivial hyperinvariant subspaces of $\mathcal{A}, M_{1}$ and $M_{2}$, other than $M$, such that $M_{1} \subseteq M \subseteq M_{2}$. Then $A^{\prime}$ is completely reducible.

Proof. Choose $X_{1}$ in lat $\mathcal{A}^{\prime}$. Since $\mathcal{A}^{\prime} \supseteq A, X_{1}$ is also in lat $A$ and, since $A$ is completely reducible, there is in $X_{2} \in$ lat $\mathcal{A}$ such that $X_{1}+X_{2}=X$. We claim that $X_{2}$ is in lat $A^{\prime}$ and therefore that $X_{2}$ is the unique complement to $X_{1}$ in lat $A$.

The claim will be established by contradiction; suppose $X_{2}$ is not in lat $A^{\prime}$. Denote by $E$ the projection onto $X_{1}$ along $X_{2}$. The proof will be divided into three parts. In the first part, we shall construct two infinite sequences of pairwise orthogonal projections, $\left\{E_{n}\right\}_{n=1}^{\infty}$ in $\left(A \mid X_{2}\right)^{\prime}$ and $\left\{F_{n}\right\}_{n=1}^{\infty}$ in $\left(A \mid X_{1}\right)^{\prime}=A^{\prime} \mid X_{1}$, and a sequence $\left\{T_{n}\right\}_{n=1}^{\infty}$ in $\operatorname{int}_{E} A$ such that $E_{n} T_{n} F_{n} \neq 0$ for all $n$.

Note that for $T \in \operatorname{int}_{E} A, B \in \mathcal{A}^{\prime} \mid X_{1}$, and $C \in\left(\mathcal{A} \mid X_{2}\right)^{\prime}, B T C \in \operatorname{int}_{E} \mathcal{A}$. For an arbitrary projection $G$ in $\mathcal{L}\left(X_{2}\right)$ let $M(G)$ denote the subspace of $X_{1}$ spanned by all $T G\left(X_{2}\right)$ with $T \in \operatorname{int}_{E} A$. Clearly, $M(G)$ is always in lat $\left(\mathcal{A}^{\prime} \mid X_{1}\right)$.

Now denote by $Y$ the intersection of the kernels of all operators in $\operatorname{int}_{E} A$. By our assumption that $X_{2}$ is not in lat $A^{\prime}$ and Lemma 3 , it follows that $Y \neq X_{2}$. On the other hand, $Y$ lies in lat $\left(A \mid X_{2}\right)^{\prime}$ and, by Lemma 4(ii), $X_{1}+Y$ lies in lat $\mathcal{A}^{\prime}$. Let $Q$ be a projection in $P\left(A \mid X_{2}\right)$ onto a subspace which is complementary to $Y$ in $X_{2}$. By hypothesis, $X_{1}+Y$ is contained in some larger nontrivial hyperinvariant subspace of $A$. By lemma 4(ii), this larger subspace has the form $X_{1}+Y+E_{1}\left(X_{2}\right)$ for some nonzero $E_{1} \in \mathcal{P}\left(A \mid X_{2}\right), E_{1} \leq Q, E_{1} \neq Q$. Repeating the same argument, one can find nonzero $E_{2} \in \mathcal{P}\left(A \mid X_{2}\right)$ with $E_{2} \leq Q-E_{1}, E_{2} \neq Q-E_{1}$. Proceeding by induction, we get an infinite sequence $\left\{E_{n}\right\}_{n=1}^{\infty}$ of pairwise orthogonal nonzero projections in $P\left(\mathcal{A} \mid X_{2}\right)$ with $E_{n} \leq Q$ for all $n$. It follows from the definition of $Q$ that $M\left(E_{n}\right) \neq 0$ for all $n$.

Now Lemma 7 provides $G_{1} \in \mathcal{P}_{0}\left(\mathcal{A} \mid X_{1}\right)$ such that $G_{1}^{\perp} M\left(E_{1}\right) \neq 0$ and $G_{1} M\left(E_{n}\right)$ $\neq 0$ for every $n$ in the infinite set $J$ of positive integers.

Renumber the elements of $J_{1}$ by $2,3, \ldots$ By Lemma 4(i), $G_{1} M\left(E_{n}\right) \in \operatorname{lat}\left(\mathcal{A} \mid X_{1}\right)^{\prime}$ for $n \geq 2$. Since

$$
\left(\left(\mathcal{A} \mid G_{1}\left(X_{1}\right)\right)^{\prime}=A \mid G_{1}\left(X_{1}\right),\right.
$$

we may again apply Lemma 7 to the algebra $A \mid G_{1}\left(X_{1}\right)$ and a sequence $G_{1} M\left(E_{2}\right)$, $G_{1} M\left(E_{3}\right), \ldots$ of its hyperinvariant subspaces. As a result, we obtain $G_{2} \in \mathcal{P}_{0}\left(\mathcal{A} X_{1}\right)$ such that $G_{2} \leq G_{1},\left(G_{1}-G_{2}\right) G_{1} M\left(E_{2}\right)=\left(G_{1}-G_{2}\right) M\left(E_{2}\right) \neq 0$, and $G_{2} G_{1} M\left(E_{n}\right)=G_{2} M\left(E_{n}\right) \neq 0$ for every $n$ from an infinite subset $J_{2}$ of $J_{1}$.

Proceeding by induction (renumbering the elements of $J_{n}$ by $n+1, n+2, \ldots$ ), we get a sequence $I=G_{0} \geq G_{1} \geq G_{2} \geq \cdots \geq G_{n} \geq \cdots$, where $G_{n} \in \mathcal{P}_{0}\left(A \mid X_{1}\right)$ and

$$
\left(G_{n-1}-G_{n}\right) M\left(E_{n}\right) \neq 0, \quad n=1,2, \ldots
$$

Let $F_{n}=G_{n-1}-G_{n}, n=1,2, \ldots$ The $F_{n}$ 's are pairwise orthogonal projections in $P\left(A \mid X_{1}\right)$, and $F_{n} M\left(E_{n}\right) \neq 0$ for $n=1,2, \ldots$ Finally, from the definition of $M\left(E_{n}\right)$, it follows that there is a sequence $\left\{T_{n}\right\}_{n=1}^{\infty}$ in $\operatorname{int}_{E} A$ such that $F_{n} T_{n} E_{n} \neq 0$ for all $n$.

In the second part of the proof we will construct a closed unbounded linear transformation $T$ defined on the linear manifold $D \subseteq X_{1}$, with range in $X_{2}$, such 
that its graph $\{T x+x, x \in D\}$ is invariant under $A$. For this, define an operator $T_{0} \in \mathcal{L}\left(X_{2}, X_{1}\right)$ as follows:

$$
T_{0}=\sum_{n=1}^{\infty} 2^{-n}\left\|F_{n} T_{n} E_{n}\right\|^{-1} F_{n} T_{n} E_{n},
$$

where the series converges in the sense of the norm in $\mathcal{L}\left(X_{2}, X_{1}\right)$. It is easy to see that $T_{0} \in \operatorname{int}_{E} A$. Now let $L=\bigcap_{n=1}^{\infty} \operatorname{Ker} F_{n}$. Then $L \in \operatorname{lat}\left(\mathcal{A} \mid X_{1}\right)$; let $N$ be in $\operatorname{lat}\left(\mathcal{A} \mid X_{1}\right)$ with $L+N=X_{1}$. Denote by $P$ the projection onto $N$ along $L$. Let us observe that for every $n$ the operator $P F_{n} \mid F_{n}\left(X_{1}\right)$ is injective; it follows that $P F_{n} T_{n} E_{n} \neq 0$ for $n \geq 1$. Now define $S \in\left(\mathcal{A} \mid X_{2}\right)^{\prime}$ as follows:

$$
S=\sum_{n=1}^{\infty} 2^{-2 n}\left\|P F_{n} T_{n} E_{n}\right\|\left\|F_{n} T_{n} E_{n}\right\|^{-1}\left\|E_{n}\right\|^{-1} E_{n}
$$

(again, the series is convergent in the sense of the norm).

We claim that the subspace

$$
M=\operatorname{cl}\left\{\left(P T_{0} x, S x\right), x \in X_{2}\right\} \subseteq X_{1} \oplus X_{2}
$$

is a graph of some linear transformation $T: D \rightarrow X_{1}, D \subseteq X_{2}$.

Indeed, the conjugate space to $X_{1} \oplus X_{2}$ is a linear space of vectors $\left(x_{1}^{*}, x_{2}^{*}\right)$, $x_{i}^{*} \in X_{i}^{*}$, endowed with the norm

$$
\left\|\left(x_{1}^{*}, x_{2}^{*}\right)\right\|=\sup \left(\left\|x_{1}^{*}\right\|,\left\|x_{2}^{*}\right\|\right) .
$$

It is easy to see that $\left(x_{1}^{*}, x_{2}^{*}\right) \in M^{\perp}$ if and only if $T_{0}^{*} P^{*} x_{1}^{*}+S^{*} x_{2}^{*}=0$. Note that, by the definition of $L$,

$$
\begin{aligned}
\operatorname{weak}^{*} \operatorname{cl}\left(\bigvee_{n=1}^{\infty} F_{n}^{*}\left(X_{1}^{*}\right)\right) & =\text { weak }^{*} \mathrm{cl}\left(\bigvee_{n=1}^{\infty}\left(\operatorname{Ker} F_{n}\right)^{\perp}\right) \\
& =\left(\bigcap_{n=1}^{\infty} \operatorname{Ker} F_{n}\right)^{\perp}=L^{\perp}
\end{aligned}
$$

Note also that $L^{\perp}=P^{*}\left(X_{1}^{*}\right), N^{\perp}=\operatorname{Ker} P^{*}$, and $L^{\perp}+N^{\perp}=X_{1}^{*}$. Let

$$
\mathcal{L}=\bigcup_{n=1}^{\infty}\left(\sum_{i=1}^{n} F_{i}^{*}\left(X_{1}^{*}\right)\right)
$$

Clearly, $\mathcal{L}$ is weak $^{*}$ dense in $L^{\perp}$. Now, for $m \geq 1$,

$$
\begin{aligned}
T_{0}^{*} P^{*} F_{m}^{*}=T_{0}^{*} F_{m}^{*} & =\left(\sum_{n=1}^{\infty} 2^{-n}\left\|F_{n} T_{n} E_{n}\right\|^{-1} E_{n}^{*} T_{n}^{*} F_{n}^{*}\right) F_{m}^{*} \\
& =2^{-m}\left\|F_{m} T_{m} E_{m}\right\|^{-1} E_{m}^{*} T_{m}^{*} F_{m}^{*}
\end{aligned}
$$

Similarly, we conclude that $E_{m}^{*}\left(X_{2}^{*}\right)$ is contained in the range of $S^{*}$ for $m \geq 1$. It follows that for each $x_{1}^{*} \in \mathcal{L}+N^{\perp}$ there exists $x_{2}^{*} \in X_{2}^{*}$ such that $T_{0}^{*} P^{*} x_{1}^{*}+S^{*} x_{2}^{*}=0$ (if $x_{1}^{*} \in N^{\perp}$, then $P^{*} x_{1}^{*}=0$ and we can take $x_{2}^{*}=0$ ), or $\left(x_{1}^{*}, x_{2}^{*}\right) \in M^{\perp}$. Now suppose $(x, 0) \in M$ for some $x \in X_{1}$. Then, for each $x_{1}^{*} \in \mathcal{L}+N^{\perp}, x_{1}^{*}(x)=0$, and, since $\mathcal{L}+N^{\perp}$ is weak* dense in $X_{1}^{*}, x=0$. This proves our claim. 
Now we shall show that $T$ is unbounded. Indeed, for every $m \geq 1, E_{m}\left(X_{2}\right) \subseteq$ $S\left(X_{2}\right)$ and therefore $E_{m}\left(X_{2}\right) \subseteq D$. Furthermore, $T S E_{m}=P T_{0} E_{m}$, or

$$
\begin{aligned}
T\left(2^{-2 m}\left\|P F_{m} T_{m} E_{m}\right\|\right. & \left.\left\|F_{m} T_{m} E_{m}\right\|^{-1}\left\|E_{m}\right\|^{-1}\right) E_{m} \\
& =2^{-m}\left\|F_{m} T_{m} E_{m}\right\|^{-1} P F_{m} T_{m} E_{m} .
\end{aligned}
$$

Since $P F_{m} T_{m} E_{m} \neq 0$,

$$
T E_{m}=2^{m}\left\|E_{m}\right\|\left\|P F_{m} T_{m} E_{m}\right\|^{-1} P F_{m} T_{m} E_{m} .
$$

Hence, $\left\|T E_{m}\right\|=2^{m}\left\|E_{m}\right\|$, which proves that $T$ is unbounded.

Now let $M_{0}$ be the closure of $\left\{P T_{0} x+S x, x \in X_{2}\right\}$ in $X$. Lemma 2 allows us to identify $M_{0}$ with $M$. That is, we may suppose that $M_{0}=\{T x+x, x \in D\}$; in particular, $M_{0} \cap X_{1}=0$ and $\left(M_{0}+X_{1} \cap X_{2}=D\right.$. For each $A$ in $A, A=$ $E A E+E^{\perp} A E^{\perp}$; it follows, since $P T_{0} \in \operatorname{int}_{E} \mathcal{A}$ and $S \in\left(\mathcal{A} \mid X_{2}\right)^{\prime}$, that for $x \in X_{2}$,

$$
\begin{aligned}
A\left(P T_{0} x+S x\right) & =\left(E A E+E^{\perp} A E^{\perp}\right)\left(P T_{0} x+S x\right) \\
& =E A E P T_{0} x+E^{\perp} A E^{\perp} S x=P T_{0} E^{\perp} A E^{\perp} x+S E^{\perp} A E^{\perp} x,
\end{aligned}
$$

which shows that $M_{0} \in$ lat $\mathcal{A}$. This ends the second part of the proof.

In the last part of the proof we obtain a contradiction. To do this, it would suffice to refer to a simple result of Fong [1], but we prefer to give a direct proof.

Since $M_{0} \in$ lat $A$ and $A$ is completely reducible, one can find $M_{1} \in$ lat $A$ such that $M_{0}+M_{1}=X$. Let $E_{0}$ denote the projection onto $M_{0}$ along $M_{1}$. Then $E_{0} \in A^{\prime}$ and hence $X_{1}$ is invariant under $E_{0}$. This implies that

$$
X_{1}=X_{1} \cap M_{0}+X_{1} \cap M_{1} \text {. }
$$

However, as we have seen, $X_{1} \cap M_{0}=0$; that means $X_{1} \subseteq M_{1}$. From the fact that $M_{0}+M_{1}=X$ and Lemma 2, it follows that the manifold $M_{0}+X_{1}$ is closed. Then $D=\left(M_{0}+X_{1}\right) \cap X_{2}$ is also closed. But $D$ is the domain of definition for a closed unbounded transformation $T$, and, by the Closed Graph Theorem, cannot be closed. This contradiction completes the proof of the theorem.

THEOREM 9. Let $A \subseteq \mathcal{L}(X)$ be a commutative unital weakly closed completely reducible algebra. Suppose that the intersection of the kernels of all compact oper. ators in $A^{\prime}$ is zero and the subspace spanned by ranges of all compact operators in $A^{\prime}$ is $X$. Then $A$ is generated, as a uniformly closed algebra, by a complete bounded totally atomic Boolean algebra of projections; in particular, $A$ is an algebra of scalar type spectral operators. Furthermore, $\mathcal{A}$ is reflexive and admits spectral synthesis.

ProOF. Let $X_{1}$ denote the subspace spanned by all one-dimensional subspaces in lat $A$, and let $X_{2}$ be a complement to $X_{1}$ in lat $A$. By Lemma $6, X_{1}$ and $X_{2}$ are in lat $A^{\prime}$. We shall show that for $A \mid X_{2}$ the conditions of the previous theorem are satisfied. Denote by $C$ the family of all compact operators in $\left(A \mid X_{2}\right)^{\prime}$. Clearly, intersections of the kernels of all operators in $C$ is zero, and the subspace spanned by all their ranges is $X_{2}$. Let $M$ be a nonzero subspace in lat $\left(\mathcal{A} \mid X_{2}\right)^{\prime}$ and $E$ be in $\mathcal{P}_{0}\left(\mathcal{A} \mid X_{2}\right)$ with $E\left(X_{2}\right)=M$. Then there is $K_{1} \in C$ such that $K_{1} \mid M \neq 0$ and (note that $M$ is infinite-dimensional), by Lomonosov's theorem [3], there is a nonzero $M_{1} \subseteq M$ such that $M_{1} \in \operatorname{lat}\left(A \mid X_{2}\right)^{\prime}$ and $M_{1} \neq M$. On the other hand, there exists $K_{2} \in C$ such that $E^{\perp} K_{2} E^{\perp} \neq 0$, for otherwise $E^{\perp} K E=E^{\perp} K E^{\perp}=0$ for each $K$ in $C$, hence $K=E K$ and $K\left(X_{2}\right) \subseteq E\left(X_{2}\right)$, which contradicts our 
hypothesis. Again, by Lomonosov's theorem, the algebra $A \mid E^{\perp}\left(X_{2}\right)$ has a nontrivial hyperinvariant subspace, and now Lemma 4(ii) implies that $A \mid X_{2}$ has a nontrivial hyperinvariant subspace $M_{2}$ strictly containing $M$. So the conditions of Theorem 8 are satisfied for $A \mid X_{2}$.

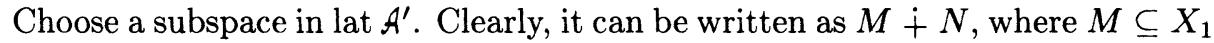
and $N \subseteq X_{2}$. By Lemma 5 , there exists $M_{1}$ in lat $\left(A \mid X_{1}\right)^{\prime}$ such that $M+M_{1}=X_{1}$, and, by Theorem 8 , we can find $N_{1} \in \operatorname{lat}\left(\mathcal{A} \mid x_{2}\right)^{\prime}$ such that $N+N_{1}=X_{2}$. But then $M_{1} \dot{+} N_{1}$ lies in lat $A^{\prime}$ and $(M+N) \dot{+}\left(M_{1} \dot{+} N_{1}\right)=X$. It follows that $A^{\prime}$ is completely reducible. Now, to conclude the proof of the theorem, it suffices to apply Theorem 1 .

The following corollary follows immediately from Theorem 9 .

COROLLARY 10. Let $T \in \mathcal{L}(X)$ be a completely reducible operator. If $T K=$ $K T$, where $K$ is an injective compact operator such that $\operatorname{cl} K(X)=X$, then $T$ is a scalar type spectral operator, and spectral synthesis holds for $T$.

COROLlaRY 11. Let $A \subseteq \mathcal{L}(X)$ be a commutative unital weakly closed completely reducible algebra. If the intersection of the kernels of all the compact operators in $A$ is zero, or if the ranges of all the compact operators in $A$ span $X$, then the conclusions of Theorem 9 hold for $A$.

PROOF. We shall show that the assumpition about the kernels is equivalent to that about the ranges. Then the result would be an immediate consequence of Theorem 9. Let $M$ be intersection of the kernels of all compact operators in $A$.

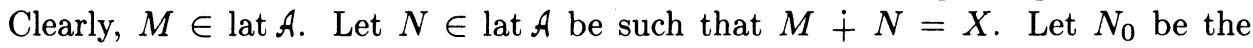
subspace spanned by all $K(N)$, where $K$ runs over the set of all compact operators in $\mathcal{A}$. Obviously, $N_{0} \in$ lat $\mathcal{A}$ and $N_{0} \subseteq N$. Let $N_{1} \in$ lat $\mathcal{A}, N_{0}+N_{1}=N$. Then, by the definition of $N_{0}$, all the compact operators in $\mathcal{A}$ vanish on $N_{1}$; that is, $N_{1} \subseteq M \cap N=0$ and $N_{0}=N$. On the other hand, the range of every compact operator in $A$ is contained in $N$. It follows that the subspace spanned by the ranges of the compact operators in $A$ is exactly $N$. But $M=0$ implies $N=X$, and vice versa.

To end this paper, we give a characterization of completely reducible compact operators.

COROLLARY 12. Every compact, completely reducible operator $K \in \mathcal{L}(X)$ is a scalar type spectral operator.

ProOF. It suffices to note that $\operatorname{Ker} K \dot{+} \operatorname{cl} K(X)=X[\mathbf{1}]$.

The author is grateful to Victor Sul'man for some helpful discussions.

\section{REFERENCES}

1. C.-K. Fong, Operator algebras with complemented invariant subspace lattices, Indiana Univ. Math. J. 26 (1977), 1045-1056.

2. A. I. Loginov and V. S. Sul'man, On reductive operators and operator algebras, Izv. Akad. Nauk SSSR Ser. Math. 40 (1976), 845-854. (Russian)

3. V. J. Lomonosov, Invariant subspaces for the family of operators which commute with a completely continuous operator, Funct. Anal. Appl. 7 (1973), 213-214.

4. Sh. Rosenoer, Completely reducible operator algebras and spectral synthesis, Canad. J. Math. (to appear). 
5. P. Rosenthal, On commutants of reductive operator algebras, Duke Math. J. 41 (1974), 829-834.

6. P. Rosenthal and A. Sourour, On operator algebras containing cyclic Boolean algebras, Pacific J. Math. 70 (1977), 243-252.

7. 16 (1977), 501-506.

Bol'Shaya Serpuhovskaya Str., 31, Korpus 6, APt. 229A, Moscow 113093, U.S.S.R. 